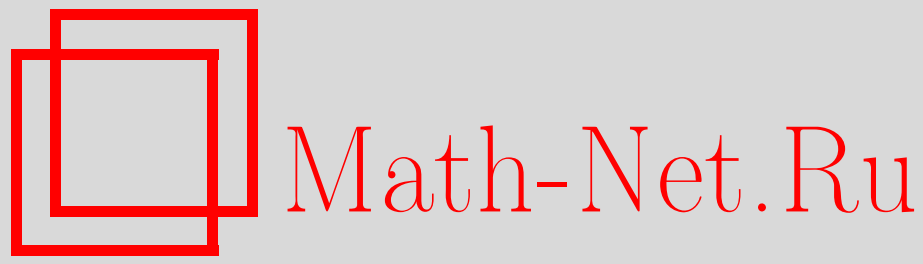

Р. С. Исмагилов, К задаче Паули, Функи. анализ и его прил., 1996, том 30, выпуск 2, 82-84

DOI: https://doi.org/10.4213/faa526

Использование Общероссийского математического портала MathNet.Ru подразумевает, что вы прочитали и согласны с пользовательским соглашением

http://www . mathnet.ru/rus/agreement

Параметры загрузки:

IP : 3.81 .55 .215

26 апреля 2023 г., 17:05:46

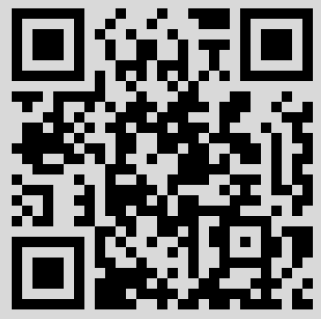


УДК 517.986.4

\section{К задаче Паули}

\section{(c) 1996. Р. С. ИСМАГИлов ${ }^{1}$}

Пусть, как обычно, $L_{2}\left(\mathbb{R}^{n}\right)$ означает комплексное гильбертово пространство функций, квадратично интегрируемых по мере Лебега в $\mathbb{R}^{n}$. Через $\widehat{f}$ обозначим преобразование Фурье элемента $f \in L_{2}\left(\mathbb{R}^{n}\right)$, определенное формулой

$$
\widehat{f}(s)=\int_{\mathbb{R}^{n}} f(x) \exp (-2 \pi i(x, s)) d x .
$$

Задача Паули, изучавшаяся в недавней работе Б. З. Мороза и А. М. Переломова [1], состоит в следующем:

описать линейно независимье пары элементов $f_{1}, f_{2}$ из $L_{2}\left(\mathbb{R}^{n}\right)$, удовлетворяющие условиям

$$
\begin{array}{ll}
\left|f_{1}(x)\right|=\left|f_{2}(x)\right| & \forall x \in \mathbb{R}^{n} \bmod 0, \\
\left|\widehat{f}_{1}(s)\right|=\left|\widehat{f}_{2}(s)\right| & \forall s \in \mathbb{R}^{n} \bmod 0
\end{array}
$$

(запись $\mathbb{R}^{n} \bmod 0$ означает подмножество пространства $\mathbb{R}^{n}$, имеющее полную лебегову меру). В [1] приведены также некоторые модификации этой задачи.

В предлагаемой заметке содержатся два результата. Во-первых, для $n=1$ мы приводим контрпример к гипотезе, сформулированной в [1]. (Эта гипотеза предлагала полное решение задачи Паули для $n=1$.) Этот контрпример показывает, что множество пар элементов со свойством (1) весьма велико (и в настоящий момент не обозримо). Во-вторых, в случае произвольного $n \geqslant 1$ мы привлекаем представление Вейля симплектической группы $\operatorname{Sp}(2 n)$ к рассматриваемому вопросу.

1. Случай $n=1$; контрпример. При $n=1$ имеется следующий простой способ построения пар $f_{1}, f_{2}$, удовлетворяющих условию (1). Возьмем точку $a \in \mathbb{R}$ и вещественные измеримые функции $\rho(x), \varphi(x), x \in \mathbb{R}$, и положим

$$
f_{1}(x)=\rho(x) \exp (i \varphi(x)), \quad f_{2}(x)=\rho(x) \exp (-i \varphi(a-x)) .
$$

Если $\rho \in L_{2}(\mathbb{R}), \rho \neq 0, \rho(x) \geqslant 0, \rho(x)=\rho(a-x)$ при $x \in \mathbb{R} \bmod 0$, то функции (2) удовлетворяют условию (1). Если, кроме того, функция $\exp (\varphi(x)+$ $\varphi(a-x))$ не постоянна, то $f_{1}, f_{2}$ линейно независимы.

В работе [1] была высказана следующая гипотеза: при $n=1$ описанная конструкция дает полное решение задачи Паули. Сейчас мы построим контрпример к этой гипотезе.

Зафиксируем вещественные бесконечно дифференцируемые функции $\varphi(x)$, $w(x), x \in \mathbb{R}$, удовлетворяющие следующим условиям:

${ }^{1}$ Работа выполнена при поддержке Фонда Дж. Сороса и Американского математического общества. 
1) $\varphi(x+1)=\varphi(x)$ для всех $x \in \mathbb{R}$, причем число 1 является наименьшим периодом функции $\varphi(x)$;

2) функция $w(x)$ неотрицательна, отлична от тождественного нуля, быстро стремится к нулю при $|x| \rightarrow \infty$ и $\widehat{w}(s)=0$ при $|s|>1 / 2$ (через $\widehat{w}(s)$ обозначено преобразование Фурье функции $w(x))$. Рассмотрим семейство функций

$$
f_{a}(x)=w(x) \exp (i \varphi(x-a)), \quad x \in \mathbb{R}, a \in(0,1 / 2) .
$$

Ясно, что любая пара функций этого семейства линейно независима и $\left|f_{a}(x)\right|=$ $w(x)$. Таким образом, функции $x \mapsto\left|f_{a}(x)\right|$ не зависят от $a$. Покажем, что функции $s \mapsto\left|\widehat{f}_{a}(s)\right|$ также не зависят от $a$.

С этой целью разложим функцию $\exp (i \varphi(x))$ в ряд Фурье:

$$
\exp (i \varphi(x))=\sum_{k=-\infty}^{\infty} c_{k} \exp (2 \pi i k x) .
$$

Отсюда получаем, что

$$
\begin{aligned}
& f_{a}(x)=\sum_{k=-\infty}^{\infty} c_{k} \exp (-2 \pi k a i) \exp (2 \pi i k x) w(x), \\
& \widehat{f}_{a}(s)=\sum_{k=-\infty}^{\infty} c_{k} \exp (-2 \pi i k a) \widehat{w}(s-k) .
\end{aligned}
$$

Так как функция $\widehat{w}(s)$ сосредоточена в отрезке $[-1 / 2,1 / 2]$, то носители функций $\widehat{w}(s-k)$ попарно не пересекаются. Следовательно,

$$
\left|\widehat{f}_{a}(s)\right|=\sum_{k=-\infty}^{\infty}\left|c_{k}\right| \cdot|\widehat{w}(s-k)| .
$$

Обозначив последнюю функцию через $v(s)$, приходим к равенствам

$$
\left|f_{a}(x)\right|=w(x), \quad\left|\widehat{f}_{a}(s)\right|=v(s) \quad \forall x \in \mathbb{R}, \forall s \in \mathbb{R}, \forall a \in(0,1 / 2) .
$$

Итак, множество $\left\{f \in L_{2}(\mathbb{R}):|f(x)|=w(x),|\widehat{f}(s)|=v(s), x, s \in \mathbb{R} \bmod 0\right\}$ содержит бесконечно много попарно не пропорциональных элементов. Это и есть контрпример к гипотезе из [1].

2. Применение представления Вейля. Пусть теперь $n \geqslant 1$. Несколько изменим постановку задачи Паули. Возьмем измеримые комплекснозначные функции $\alpha(x), \beta(s), x, s \in \mathbb{R}^{n}$, такие, что $|\alpha(x)|=1,|\beta(s)|=1$ при $x \in \mathbb{R}^{n} \bmod 0$, $s \in \mathbb{R}^{n} \bmod 0$. Рассмотрим следующую задачу:

описать линейно независимые пары элементов $f_{1}, f_{2} \in L_{2}\left(\mathbb{R}^{n}\right)$, такие, umo

$$
\begin{array}{ll}
f_{2}(x)=\alpha(x) f_{1}(x) & \forall x \in \mathbb{R}^{n} \bmod 0, \\
\widehat{f}_{2}(s)=\lambda \beta(s) \widehat{f_{1}}(s) & \forall s \in \mathbb{R}^{n} \bmod 0
\end{array}
$$

для некоторого числа $\lambda,|\lambda|=1$. (Функции $\alpha(x), \beta(s)$ можно назвать фазовыми.) 
Решим эту задачу для функций

$$
\alpha(x)=\exp (\pi i(a x, x)), \quad \beta(s)=\exp (\pi i(b s, s)),
$$

где $a, b$ - симметрические вещественные матрицы порядка $n, \mathrm{a}(\cdot, \cdot)$ обычное скалярное произведение в $\mathbb{R}^{n}$.

С этой целью рассмотрим симплектическую группу

$$
\begin{gathered}
G=\operatorname{Sp}(2 n)=\left\{g=\left(\begin{array}{ll}
a & b \\
c & d
\end{array}\right), a, b, c, d \in \operatorname{Mat}_{n}(\mathbb{R}), g^{\prime} J g=J\right\}, \\
J=\left(\begin{array}{cc}
0 & -I \\
I & 0
\end{array}\right) .
\end{gathered}
$$

(Здесь $g^{\prime}$ - транспонированная к матрице $g, I$ - единичная матрица.) В пространстве $L_{2}\left(\mathbb{R}^{n}\right)$ действует проективное представление Вейля $T(g), g \in G$, определенное равенствами [2, с. 43$]$

$$
\left(T\left(g_{b}\right) f\right)(x)=\exp (\pi i(b x, x)), \quad g_{b}=\left(\begin{array}{cc}
I & b \\
0 & I
\end{array}\right), b=b^{\prime}, \quad(T(J) f)(x)=\widehat{f}(x) .
$$

Условия (3) равносильны равенствам $f_{2}=T\left(g_{a}\right) f_{1}, T(J) f_{2}=\lambda T\left(g_{b}\right) T(J) f_{1}$. Отсюда $T\left(J^{-1} g_{-b} J g_{a}\right) f_{1}=\lambda_{1} f_{1},\left|\lambda_{1}\right|=1$. Положим $h_{a, b}=J^{-1} g_{-b} J g_{a}$. Тогда

$$
h_{a, b}=\left(\begin{array}{cc}
I & a \\
b & I+b a
\end{array}\right), \quad T\left(h_{a, b}\right) f_{1}=\lambda_{1} f_{1} .
$$

Итак, $f_{1}$ - собственный вектор для $T\left(h_{a, b}\right)$. Но это возможно лишь тогда, когда матрица $h_{a, b}$ сопряжена (в группе $G$ ) ортогональной матрице вида

$$
\left(\begin{array}{cc}
c & d \\
-d & c
\end{array}\right), \quad c, d \in \operatorname{Mat}_{n}(\mathbb{R}), c+i d \in U(n) .
$$

Автору неизвестна полная характеризация пар симметрических матриц $a, b$, для которых выполнено это условие. Легко доказать, что оно выполняется, если $a>0,-4 a^{-1}<b<0$ (неравенства для матриц определяются через квадратичные формы). В этом достаточном условии можно также поменять местами матрицы $a$ и $b$. При указанных условиях существует пара $f_{1}, f_{2}$ со свойством (3). При $n=1$ эта пара может быть записана в виде (2). Мы не останавливаемся на явных формулах для функций $f_{1}, f_{2}$ из (3). Заметим лишь, что они выражаются через функции Эрмита и экспоненты.

\section{ЛиТЕРАТУРА}

1. Мороз Б. З., Переломов А. М. Теор. матем. физ., 101, №1, 60-65 (1994). 2. Лион Ж., Вернь М. Представление Вейля, индекс Маслова и тэта-ряды. Мир, M., 1983. 